\title{
Estudios clínicos sobre la enfermedad celíaca (2014-2019): revisión sistemática de la prevalencia de la presentación clínica y enfermedades asociadas por edades
}

\author{
Marta Miró ${ }^{\mathrm{a}}$, Manuel Alonso-Garrido ${ }^{\mathrm{a}}$, Manuel Lozano ${ }^{\mathrm{a}}$, Lara Manyes ${ }^{\mathrm{a}, *}$ \\ a Departamento de Medicina Preventiva y Salud Pública, Ciencias de la Alimentación, Toxicología y Medicina Legal, \\ Universitat de València, Valencia, España. \\ *Iara.manyes@uv.es
}

Editor Asignado: Rafael Almendra-Pegueros. Universidad Autónoma de San Luis Potosí. San Luis Potosí, México.

Recibido el 30 de enero de 2020; aceptado el 12 de abril de 2020; publicado el 20 de abril de 2020.

\section{PALABRAS CLAVE \\ Enfermedad Celíaca; \\ Prevalencia; \\ Signos y Síntomas; \\ Enfermedades \\ Autoinmunes.}

Estudios clínicos sobre la enfermedad celíaca (2014-2019): revisión sistemática de la prevalencia de la presentación clínica y enfermedades asociadas por edades

\section{RESUMEN}

Introducción: La enfermedad celíaca (EC) se caracteriza por una gran variedad de signos, síntomas y enfermedades asociadas en su forma de presentación, incluso puede cursar de forma asintomática. Recientes estudios muestran la variación del espectro clínico según la edad. En niños y niñas pequeñas predomina la forma clásica con síntomas como distensión abdominal, disminución del apetito, diarrea y pérdida de peso. Las manifestaciones atípicas frecuentes en niños mayores son dolor abdominal, estreñimiento, reflujo, vómitos, fatiga, talla baja. En adultos se observa una reducción de la forma de presentación clásica e incremento de la no clásica.

Material y Métodos: Se realizó una revisión sistemática en la que se han incluido los artículos sobre signos y síntomas de la EC clásica y atípica en niños y adultos, además de las enfermedades que se asocian de forma frecuente a esta patología. Las bases de datos utilizadas para la búsqueda de los artículos fueron PubMed, Web of Science y Scopus, de los últimos seis años (2014-2019). Se han evaluado un total de 164 artículos tras los criterios de selección, de los cuales se han incluido en el estudio los 20 artículos más relevantes.

Resultados: La EC se diagnostica con mayor frecuencia en mujeres y a edades más tempranas que a los hombres. En niños menores de dos años predomina la EC clásica, aunque de forma más intensa que en niños y niñas pequeñas. En niños y niñas mayores, adolescentes y edad adulta varía la forma de presentación hacia la atípica o no clásica y asintomática.

Conclusiones: El incremento en la prevalencia en los últimos años, gran heterogeneidad de síntomas, enfermedades asociadas y la variación del espectro clínico hacia una forma atípica con síntomas extraintestinales causa retraso en su diagnóstico por lo que deben reconocerse para que se detecte de manera más precoz. 
Clinical studies on celiac disease (2014-2019): systematic review about the age ranged prevalence of clinical presentation and associated diseases

\section{KEYWORDS}

Celiac Disease;

Prevalence;

Signs and

Symptoms;

Autoimmune

Diseases.

\section{ABSTRACT}

Introduction: Celiac disease (CD) is characterized by a wide variety of signs, symptoms and associated diseases in its presentation, it can even be asymptomatic. Recent studies show the variation of the clinical spectrum according to age. In young children, classic celiac disease predominates with symptoms such as abdominal distension, decreased appetite, diarrhea and weight loss. Frequent atypical manifestations in older children are abdominal pain, constipation, reflux, vomiting, fatigue and short stature. In adults there is a reduction in the classical presentation and an increase in the non-classical.

Material and Methods: A systematic review was conducted in which articles on signs and symptoms of classical and atypical CD in children and adults have been included, in addition to the diseases that are frequently associated with this pathology. The databases used to search the articles were PubMed, Web of Science and Scopus, from the last six years (2014-2019). A total of 164 articles have been evaluated following the selection criteria, of which the 20 most relevant articles have been included in the study.

Results: CD is diagnosed more frequently in women and at earlier ages than men. In children under two years old, classical CD predominates although more intensely than in young children. In older children, teenagers and adults, the form of presentation changes towards atypical or non-classical and asymptomatic.

Conclusions: The increased prevalence in the last years, great heterogeneity of symptoms, associated diseases and the variation of the clinical spectrum towards an atypical form with extraintestinal symptoms causes delay in its diagnosis so they must be recognized to be detected earlier.

\section{CITA}

Miró M, Alonso-Garrido M, Lozano M, Manyes L. Estudios clínicos sobre la enfermedad celíaca (2014-2019): revisión sistemática de la prevalencia de la presentación clínica y enfermedades asociadas por edades. Rev Esp Nutr Hum Diet. 2020; 24(3): 234-46. doi: 10.14306/renhyd.24.3.998

\section{INTRODUCCIÓN}

La enfermedad celíaca (EC) es la enteropatía crónica de origen autoinmune más frecuente a nivel mundial, desencadenada por la ingestión de gluten y proteínas relacionadas, en personas genéticamente susceptibles. Se caracteriza por la presentación conjunta de manifestaciones clínicas sistémicas, anticuerpos específicos en suero como antitransglutaminasa tisular (ATGt), antiendomisio (EMA) y péptidos deaminados de la gliadina (DPG), los cuales sustituyen a antigliadina (AGA), haplotipos HLA DQ2 y/o DQ8 y presencia de alteraciones histopatológicas en la biopsia duodenal como incremento de linfocitos intraepiteliales (LIE), atrofia vellositaria e hiperplasia de las criptas ${ }^{1}$.
La prevalencia global de EC se estima alrededor del $1 \%^{2}$. La incidencia de EC se ha incrementado en los últimos años según la edad y área geográfica, las tasas en personas adultas han aumentado de 3,08/100.000 habitantes en el año 1995 a 11,13/100.000 habitantes en 2005 y en niños y niñas de 2,08/100.000 habitantes por año (1981-1985) a $6,89 / 100.000$ habitantes por año (2001-2005)3. En la actualidad, se están diagnosticando nuevos casos de EC entre la tercera y cuarta décadas de vida, observándose en mayor proporción en mujeres que en hombres (2-3:1) 4 . La edad media en el diagnóstico de la EC en personas adultas es de 52 y 44 años en hombres y mujeres, respectivamente ${ }^{5}$.

La EC es un trastorno multisistémico, caracterizado por manifestaciones clínicas muy variables, similares en ambos 
sexos y a todas las edades, clasificadas en los siguientes grupos según Glissen et al. (2019) ${ }^{6}$ y Kalleveen et al. (2018): 1) La EC clásica se caracteriza por presentar sintomatología digestiva, destacando la diarrea crónica, pérdida de peso, síndrome de malabsorción (esteatorrea), retraso en el desarrollo y "crisis celíacas" (diarrea, distensión abdominal, hipotensión, hipoproteinemia con alteraciones metabólicas severas), ésta última sobre todo en niños y niñas. 2) La EC no clásica o atípica se asocia tanto a manifestaciones gastrointestinales (dolor y distensión abdominal, estreñimiento, vómitos) como manifestaciones extraintestinales (anemia ferropénica, aumento de enzimas hepáticas, osteopenia y osteoporosis, artritis, aftas orales, defectos del esmalte dentario, fatiga, talla baja, diabetes mellitus tipo I (DM-I), tiroiditis autoinmune, dermatitis herpetiforme (DH), infertilidad de origen desconocido y alteraciones neurológicas). 3) En la EC asintomática no aparece ningún síntoma, pero los pacientes deben ser tratados con una dieta estricta libre de gluten, en la que se ha podido comprobar que mejora la calidad de vida. Se incluyen la EC silente, la latente y la sensibilidad al gluten no celíaca.

Es necesario el cribado de EC en manifestaciones atípicas que a menudo pueden no asociarse a la enfermedad y causan retraso en el diagnóstico. La prevalencia de EC en niños y niñas estreñidas con resistencia al tratamiento es de $1: 28^{8}$. Otra manifestación en niños es el defecto del esmalte dentario cuya prevalencia en la EC varía del $38-96 \%$ y en su mayoría el tipo es grado $1^{9}$.

Otra manifestación extraintestinal a tener en cuenta son las alteraciones neurológicas y psiquiátricas, la prevalencia entre EC y epilepsia varía del 0,8-6\% ${ }^{10}$, otras descritas son la ataxia cerebelosa, neuropatía periférica, demencia, depresión y cefaleas ${ }^{11}$.

La prevalencia de EC y anemia ferropénica varía ampliamente del 1,8-20\%, es una manifestación común de la enfermedad ${ }^{12}$.

Alrededor del 35\% de los pacientes celíacos son más susceptibles de padecer otras enfermedades autoinmunes de manera conjunta con la EC como DM-I, tiroiditis autoinmune, enfermedad inflamatoria intestinal (EII) (enfermedad de Crohn y colitis ulcerosa), artritis reumatoide, trastornos del tejido conectivo y otros trastornos hepáticos, dermatológicos y cardíacos de origen autoinmune ${ }^{13,14}$. La prevalencia informada en la literatura de EC en colitis colagenosa y linfocítica es del 3-23\% y del 6-15\%, respectivamente ${ }^{15}$. El síndrome de Down puede asociarse con otros trastornos autoinmunes y manifestarse a cualquier edad, la prevalencia de EC en sujetos con síndrome de Down varía de $4,6-13 \%{ }^{16}$.
La mejora en la sensibilidad y especificidad de las pruebas diagnósticas han Ilevado a un mayor reconocimiento de la enfermedad en la detección tanto de pacientes sintomáticos y asintomáticos, como de aquellos que pertenecen a grupos de riesgo como familiares de primer grado, DM-I, déficit selectivo de $\lg \mathrm{A}$, tiroiditis autoinmune, enfermedad de Addison y síndromes de Sjögren, Turner, Williams, Down entre otros ${ }^{17}$. Es de señalar que el riesgo de padecer EC es seis veces mayor en pacientes con síndrome de Down ${ }^{18}$. Aunque aún sigue siendo una patología infradiagnosticada, ya que se estima que por cada caso diagnosticado existen de $5-10$ casos sin diagnosticar ${ }^{19}$.

El aumento de la variabilidad en la sintomatología, grupos de riesgo y la deriva hacia formas de EC no clásica, silente o latente, hacen necesarios estudios que determinen nuevas estrategias de diagnóstico basadas en las nuevas manifestaciones clínicas de la EC y/o aquellas propias de enfermedades asociadas. La realización del estudio queda justificada por varios motivos: 1) Necesidad de informar a los profesionales de la salud para que la EC se detecte de manera más precoz, evitando continuas visitas médicas que causan retraso en el diagnóstico y costes sanitarios. 2) Necesidad del estudio de nuevas directrices para detectar a un elevado número de pacientes que son celíacos y no presentan síntomas, aunque no hay consenso entre las sociedades científicas para un cribado de la población general. 3) Al tratarse de un trastorno de origen autoinmune, los pacientes son más susceptibles de padecer otras patologías inmunitarias, por lo que es necesario reconocer a aquellos que pertenezcan a grupos de riesgo y reducir la morbimortalidad.

El objetivo de este trabajo es evaluar las variaciones de prevalencia en las formas de presentación clínica (clásica 0 atípica) de la EC y enfermedades asociadas en personas adultas y niños y niñas, ya que se observan cambios en el espectro clínico en los últimos años.

\section{$\longrightarrow$ MATERIAL Y MÉTODOS}

Para la elaboración de esta revisión sistemática se siguieron las recomendaciones propuestas por la declaración PRISMA ${ }^{20}$. Los estudios se identificaron mediante la búsqueda en las bases de datos PubMed, Web of Science y Scopus de los últimos seis años (2014-2019). La estrategia de búsqueda se basó en el uso de las siguientes palabras clave: celiac disease, sign, symptom, prevalence, autoimmune disease. 
La búsqueda inicial que incluyó los siguientes filtros: período (rango 2014-2019), sujetos humanos, estudios clínicos, ensayos clínicos, conjuntos de datos, metaanálisis, ensayos aleatorizados y ensayos controlados. Se realizaron dos búsquedas entre las tres bases de datos: 1) celiac disease AND sign AND symptom AND prevalence y 2) celiac disease AND sign AND symptom AND autoimmune disease.

\section{RESULTADOS}

\section{Selección de estudios}

Se obtuvieron un total de 769 artículos. Se eliminaron 190 artículos por estar duplicados y se excluyeron 415 artículos tras la lectura de los resúmenes por no ceñirse a la temática del trabajo planteado. Se examinaron de manera completa un total de 164 artículos y tras su lectura se descartaron 144 por Ios siguientes motivos: a) por no localizarse, bien por estar restringido su acceso o por no encontrar la página web; b) por estar en un idioma diferente al inglés o español, por no detallar formas de presentación ni enfermedades asociadas o falta de datos para nuestro estudio; d) ser artículos de revisión o casos clínicos. Finalmente, por todos los motivos expuestos anteriormente se incluyeron 20 artículos en el estudio.
En la Figura 1 se muestra el diagrama de flujo seguido en la revisión y selección de artículos.

\section{Datos extraídos de la revisión sistemática}

En esta revisión se han incluido los artículos sobre signos y síntomas de la EC clásica y atípica en niños y adultos, además de las enfermedades que se asocian de forma frecuente a esta patología. Las principales características de los 20 estudios incluidos en esta revisión se resumen en la Tabla 1. Doce estudios se realizaron en personas adultas y ocho en niños y niñas. La mayoría de trabajos considera personas adultas a las mayores de 18 años y sólo un estudio se refiere a las mayores de 15 años. En el caso de niños y niñas, la mayoría considera a las menores de 18 años y dos estudios menores de 15 años. Estos estudios han sido seleccionados por detallar formas de presentación, enfermedades asociadas, signos y síntomas de la EC en diferentes grupos de edad para observar las variaciones del espectro clínico en los últimos años.

Todos los estudios incluidos en este trabajo muestran una mayor prevalencia de EC en el sexo femenino tanto en personas adultas como menores. Las mujeres se diagnostican a edades más tempranas que los hombres, alrededor de 40 y 50 años $^{21-24}$. Se han obtenido de la Tabla 1 los siguientes resultados: total de pacientes adultos: 2.831

Figura 1. Diagrama de selección de artículos.

Número de artículos identificados en las búsquedas:

1. Celiac disease AND sign AND symptom AND prevalence

2. Celiac disease AND sign AND symptom AND autoimmune disease

PubMed: 282 WOS: 72 Scopus: 415

Artículos duplicados eliminados 190

Lectura de resúmenes de $\mathbf{5 7 9}$ artículos

Número de artículos seleccionados para lectura completa: 164

Artículos incluidos en el estudio: 20
Se han excluido 144 artículos por:

- Excluidos por no localizarse: 4 - Otro idioma distinto de inglés/español: 5 - Artículos de revisión: 29

- Faltan datos para nuestro estudio/poco específico: 106 
Tabla 1. Principales características de los artículos incluidos en esta revisión que evalúan signos, síntomas y enfermedades asociadas a la EC.

\begin{tabular}{|c|c|c|}
\hline Tipo de estudio & $\begin{array}{l}\text { N/Tipo } \\
\text { población }\end{array}$ & Objetivo \\
\hline $\begin{array}{l}\text { Cohorte, } \\
\text { retrospectivo }\end{array}$ & $\begin{array}{l}\mathrm{N}=93 \\
\text { Adultos } \\
\text { y adultas } \\
\text { celíacos ( } \geq 15 \\
\text { años hasta } \\
75 \text { años) }\end{array}$ & $\begin{array}{l}\text { Describir incidencia } \\
\text { de EC y sus } \\
\text { complicaciones en } \\
\text { el momento del } \\
\text { diagnóstico }\end{array}$ \\
\hline $\begin{array}{l}\text { Cohorte, } \\
\text { retrospectivo }\end{array}$ & $\begin{array}{l}\mathrm{N}=412 \\
\text { Adultos } \\
\text { y adultas } \\
\text { celíacos ( } \geq 18 \\
\text { años) }\end{array}$ & $\begin{array}{l}\text { Enfoque en la } \\
\text { presentación } \\
\text { de síntomas, } \\
\text { coexistencia de } \\
\text { enfermedades } \\
\text { autoinmunes y } \\
\text { complicaciones } \\
\text { malignas de la EC }\end{array}$ \\
\hline
\end{tabular}

Adultos al diagnóstico,

$\begin{array}{l:l}\text { Cohorte, } & \text { Adultos } \\ \text { retrospectivo } & \text { y adultas } \\ & \text { celíacos } \\ & (\geq 18 \text { años })\end{array}$
presentación clínica y prevalencia de enfermedades asociadas

Analizar el cambio de presentación clínica durante el período 1960-2015 (disminución EC clásica y aumento

$\begin{array}{l:l}\text { Cohorte, } & \text { Adultos } \\ \text { retrospectivo } & \text { y adultas } \\ & \text { celíacos } \\ & (18-91 \text { años })\end{array}$

EC no clásica o silente)

\section{$\mathrm{N}=341$}

$(91 \mathrm{EC}+\mathrm{EI}) \mathrm{y}$

\section{Casos y} controles $250 \mathrm{EC} \sin \mathrm{El}$ Adultos y adultas celíacos (18-76 años)

\section{$\mathrm{N}=129$}

Multicéntrico, prospectivo, búsqueda de casos

Adultos y adultas con diagnóstico de EC (16-99 años)

Evaluar el espectro de enfermedades autoinmunes $y$ factores de riesgo asociados a personas adultas celíacas

Evaluar la importancia de las diferentes formas de presentación asociadas a la EC para incrementar su detección
Resultados

Referencia/

País

Mujer 76\%. Incidencia: 6,4/100.000 personas-año Edad media en el momento del diagnóstico 36 años Síntomas: diarrea, pérdida de peso (EC clásica) en la mitad de los pacientes

Enfermedades asociadas: DM-I, disfunción tiroidea, 21 Dinamarca DH EC clásica > EC no clásica, EC silente

Mujeres el 65,6\%.

Edad media de diagnóstico: 39,45 años

$1 / 3$ presentó diarrea, fatiga, dolor abdominal,

pérdida de peso, pero están aumentando síntomas atípicos como estreñimiento y reflujo

Enfermedades asociadas (25,2\%): DM-I, colitis

microscópica, tiroiditis autoinmune

Complicaciones (12,9\%): carcinoma basocelular, EALT, colorrectal

Mayor prevalencia de EC en mujeres y se diagnostican a edades más tempranas que los hombres

Formas de presentación: EC clásica > EC no clásica > EC silente

Síntomas: mujeres (náuseas, vómitos, estreñimiento), hombres (esteatorrea).

Enfermedades autoinmunes: hipotiroidismo, colitis microscópica, Graves, psoriasis, DM-I

Prevalencia EC mujeres:hombres ratio 2.2:1

EC clásica presentación más común en el momento del diagnóstico (diarrea, esteatorrea, pérdida peso, retraso en el desarrollo)

Síntomas: mujeres (estreñimiento, distensión abdominal y anemia ferropénica), hombres (pérdida de peso)

EC clásica > EC no clásica > EC silente

Enfermedades asociadas: enfermedad tiroidea, DM-I, psoriasis, Ell

$77,4 \%$ mujeres

$26,6 \%$ de celíacos tenían al menos una El, endocrinas y dermatológicas las más frecuentes (tiroiditis

autoinmune, psoriasis, DM-I, DH)

EC no clásica $>E C$ clásica > EC silente

Factores de riesgo $(E C+E I)$ : sobrepeso, persistencia síntomas, antecedentes familiares con EI

Búsqueda de pacientes con anticuerpos ATGt y EMA Edad media 55,8 años y $65,1 \%$ mujeres

Prevalencia 3,2\% de EC

Síntomas GI (diarrea, síntomas del intestino irritable, pérdida peso) no se asociaron con la EC (EC clásica) $L a$ anemia, antecedentes enfermedades autoinmunes, osteoporosis, familiares de primer grado y edad temprana se asociaron al diagnóstico de EC (EC atípica)

EC atípica y EC silente $>$ EC clásica
Estados Unidos

24 Irlanda 
Tipo de estudio Retrospectivo

Transversal

Prospectivo

\section{Casos y} controles

\begin{tabular}{l|l} 
controles & $\begin{array}{l}(18,1-87,7 \\
\text { años })\end{array}$ \\
& \\
& \\
& \\
& \\
& \\
& \\
Cohorte, & \\
retrospectivo & $\begin{array}{l}\text { Adultos y } \\
\text { adultas } \\
(18-68 \text { años })\end{array}$
\end{tabular}

$\mathrm{N}=159$

Adultos

y adultas

celíacos

(18,1-87,7

Adultos

y adultas

(23-75 años)

$\mathrm{N}=80$

Adultos

y adultas

y edad

avanzada

NCGS

(18-65 años)

Evaluar

características

clínicas, factores

psicológicos, y

calidad de vida

relacionada con

la salud, antes

y después del

diagnóstico

Investigar la

variación del

estado nutricional

y sintomatología

en pacientes

EC y NCGS tras

asesoramiento

dietético

Evaluar la utilidad de la búsqueda de casos de EC en la población adulta no diagnosticada, indicaciones para la prueba serológica, síntomas clásicos y no clásicos

Analizar la presentación clínica, serología, alteraciones histopatológicas y datos de laboratorio en un período de 10 años (2003-2013)

\section{Resultados}

Referencia/

País

Mayor prevalencia en el sexo femenino

CoelD síntomas intestinales, enfermedades

asociadas, cribado familiar y síntomas

extraintestinales)

LymD y NorD (síndromes funcionales) síntomas

gastrointestinales, como dolor abdominal, dispepsia, pérdida de peso

Síntomas extraintestinales (anemia) y enfermedades asociadas (disfunción tiroidea) más frecuentes en CoelD Todos los pacientes de CoelD portaban haplotipo HLA DQ2/DQ8

La duodenitis linfocítica tiene una presentación clínica diferente en pacientes CoelD y en pacientes con síndrome Gl funcional

El $80 \%$ fueron mujeres, edad media 48,6 años Síntomas típicos en la mayoría de los pacientes: diarrea, distensión-dolor abdominal, fatiga, dispepsia, pérdida de peso y $30 \%$ atípicos (estreñimiento, alteraciones cutáneas, osteoporosis, infertilidad, abortos recurrentes)

EC clásica > EC no clásica (esta última en aumento) $63,8 \%$ diagnóstico previo de Ell y el 28,8\% dispepsia provoca retraso diagnóstico, peor calidad de vida

Edad media 42 años y mujeres el $80 \%$

Prevalencia EC $(66,2 \%)$ y NGGS $(33,8 \%)$

Síntomas intestinales seguidos de extraintestinales

Dolor-distensión abdominal, estreñimiento y

epigastralgia principales motivos de consulta.

Tras DSG se normalizan los síntomas tanto en EC como en NCGS

Edad media 44,2 años y $61 \%$ mujeres

Prevalencia de EC no diagnosticada $0,88 \%$

El 23\% se diagnosticó de EC con un tiempo medio de diagnóstico de 5,69 años

Síntomas asociados con el diagnóstico de EC:

déficits de vitaminas, anemia, déficit de hierro,

diarrea crónica, dolor-distensión abdominal, atrofia

vellositaria, DH, hipotiroidismo, infertilidad, fatiga,

familiares de primer grado, DM-I, vitíligo.

Personas con EC no diagnosticada son más

propensas a desarrollar osteoporosis, $\mathrm{DH}$, fatiga crónica, tiroiditis y enfermedades autoinmunes.

La estrategia de búsqueda de casos es ineficaz (tamaño de muestra 47.557)

Prevalencia de EC: 147 mujeres $(75,3 \%)$ y 48 hombres $(24,7 \%)$, edad media 33 años.

EC con síntomas GI $(67,17 \%)$ : dolor abdominal inespecífico, dispepsia, distensión abdominal, flatulencia, acidez, diarrea crónica y pérdida de peso. EC con síntomas no-GI (32,8\%): anemia ferropénica, $\mathrm{DH}$, osteoporosis, elevación enzimas hepáticas, fatiga crónica, dolor articular, cefalea, infertilidad, ataxia, epilepsia, estomatitis aftosa

Prevalencia de historia familiar de EC $(7,17 \%)$, déficit de $\lg \mathrm{A}(6,2 \%)$ y $\mathrm{EI}(3,5 \%)$

El grado de atrofia más característico fue Marsh III ( $71,05 \%$ de los pacientes) y el $75 \%$ de los pacientes dieron positivo los anticuerpos ATG (correlación estadísticamente significativa entre el grado de atrofia y el título de anticuerpos ATG)

Todos los pacientes con DH presentaron atrofia vellositaria severa y alto nivel de anticuerpos ATG
27

Italia

México

Brasil

30

Minesota

Rumanía 
Estudios clínicos sobre la enfermedad celíaca (2014-2019): revisión sistemática de la prevalencia de la presentación clínica y enfermedades asociadas por edades

Tipo de estudio

N/Tipo
población

$\mathrm{N}=73$

Transversal

Adultos y adultas (18-58 años)

$\mathrm{N}=191$

Retrospectivo

Prospectivo, observacional multicéntrico

$\mathrm{N}=974$

Niños y niñas celíacas (<15 años)

$N=165$

Retrospectivo

Niños y niñas celíacas (<18 años)

\section{$\mathrm{N}=188$}

Transversal

(111 DM-I y 77 SD) de 10 meses a 18 años de edad

$\mathrm{N}=520$

(22 DM-I

$+498$

Cohorte retrospectivo
Analizar y comparar el modo de presentación clínica y complicaciones de la EC en niños y niñas durante dos períodos (20052008 y 2008-2012)

Evaluar la incidencia y modo de presentación clínica en niñas y niños celíacos españoles

Evaluar el modo de presentación en niñas y niños celíacos de Nueva York en un período de 10 años

Investigar los síntomas GI, extraintestinales sugestivos de EC en niñas, niños y adolescentes con DM-I y SD

Evaluar si es cribado activo de clínica) Niños y niñas (0-17 años)
Determinar síntomas, patologías asociadas y características de la EC necesario el

\section{Resultados}

Referencia/

País

Prevalencia de EC: 56 mujeres $(76,7 \%)$ y 17 hombres $(23,3 \%)$, edad media $(30.15 \pm 9.64)$

La aparición de EC en $<18$ años (39,72\%) y $\geq 18$ años $(60,27 \%)$

El 38,29\% tienen bajo peso; el 8,51\% sobrepeso y

$2,12 \%$ obesidad

El 20,5\% pacientes con historia familiar de EC

Síntomas GI (1,40\%): diarrea $(69,2 \%)$ y dolor

abdominal $(53,8 \%)$; síntomas extra-digestivos (41,1\%)

y síntomas extraintestinales $(4,1 \%)$ en los que incluye astenia $(67,9 \%)$ y pérdida de peso $(53,8 \%)$ y en menor proporción dolor óseo y articular, cefalea, ansiedad, edema, retraso crecimiento, hipoplasia esmalte dentario, entre otros.

El 7,7\% presentó hipocalcemia.

Patologías asociadas: anemia (43,6\%), trastorno nervioso $(8,21 \%)$, úlceras bucales $(5,1 \%)$

Edad media de diagnóstico 8,1 años, más de la mitad fueron niñas.

EC clásica (grupo 1) y EC no clásica (grupo 2)

EC no clásica > EC silente $>$ EC clásica

Síntomas frecuentes: diarrea crónica, dolor

abdominal, distensión abdominal, talla baja, anemia ferropénica, retraso en el desarrollo, alteraciones del metabolismo óseo, en ambos grupos

Aumento de prevalencia de EC en DM-I, tiroiditis, SD,

déficit selectivo de IgA y familiares de primer grado

Más de la mitad fueron niñas, edad media de diagnóstico 2,3 años

Incidencia: 7,9 casos $/ 1.000$ nacidos vivos y 54 casos/100.000 personas año

EC clásica $>E C$ no clásica $>E C$ silente

EC clásica en el grupo 0-2 y >2-6 años y la EC no

clásica y asintomática en niños $>6$ años

Enfermedades asociadas: déficit lgA, DM-I, tiroiditis, SD

32

Argelia

oriental

Prevalencia de EC mujeres:hombres (99:76). Edad media de diagnóstico 10,7 años y la mayoría con EC no clásica

Forma de presentación por orden de frecuencia: dolor abdominal, estreñimiento, diarrea, familiares de

primer grado, pérdida de peso, retraso del desarrollo, reflujo, vómitos, erupción cutánea, fatiga, talla baja, enfermedad tiroidea y SD

Edad media 8,95 años y el $54,3 \%$ mujeres Prevalencia de EC en DM-I es $4,5 \%$ y en SD de $13 \%$ $53,7 \%$ síntomas GI (DM-I 46,8\%, SD 63,6\%) y $16 \%$ extraintestinales.

Anticuerpos ATGt 28,2\%

EC GI $(32,5 \%)$ la mayoría en SD, silente (5\%) en DM-I y potencial $(62,5 \%)$ en la mayoría de DM-I

Edad media de diagnóstico: 7,8 años y 63,8\% fueron niñas

Grupo de sospecha clínica (61,3\% síntomas Gl y el $29,5 \%$ manifestaciones extraintestinales)

Alrededor $1 / 2$ niños con DM-I en el momento del diagnóstico de EC presentaron atrofia vellositaria con DM-I moderada-severa

La EC se asoció con mayor frecuencia al déficit $\lg \mathrm{A}$, tiroiditis autoinmune, SD 
Estudios clínicos sobre la enfermedad celíaca (2014-2019): revisión sistemática de la prevalencia de la presentación clínica y enfermedades asociadas por edades

Tipo de estudio

Prospectivo

Retrospectivo

$\mathrm{N}=400$

Niños y niñas

celíacas

(0-14,9 años)

$\mathrm{N}=1.030$

Niños y niñas

celíacas

(<18 años)

período de

1973-2013

Evaluar la edad al diagnóstico, síntomas clínicos y resultados de la biopsia en tres períodos de tiempo

Incidencia de EC en niños y niñas noruegas entre el período 2000 al 2010, variación de síntomas y cambios histopatológicos en biopsia duodenal

Comparar la

$\mathrm{N}=54$

Transversal frecuencia y las características

clínicas de la

presentación diarreica versus no diarreica de la EC
Resultados

Referencia/

País

Edad media de diagnóstico 5,7-6,3 años, mujeres:hombres (1.8:1)

3 grupos de estudio (1973-1983, 1984-1996, $1997-$

2013) y 3 subgrupos: edad media pre-epidémico $(2,2$ años), epidémico ( 2,8 años), post-epidémico $(8,2$ años)

Síntomas Gl y retraso desarrollo/talla baja frecuentes en los 2 primeros períodos (con síntomas más severos) seguidas de síntomas extraintestinales, los pacientes asintomáticos (19\%) frecuentes en el tercer período y con síntomas menos graves

Disminución de síntomas en los 3 períodos conforme aumenta la edad, siendo menos severos

Síntomas Gl, retraso desarrollo/talla baja (01,9 años), extraintestinales (15-17,9 años) y asintomáticos/grupos de riesgo (5-14,9 años) Se ha incrementado la edad media del diagnóstico de EC

Prevalencia EC mujeres:hombres ratio 1.5:1

Incidencia 2000-2002: 15.9 casos/100.000 personas año y del 2008-2010 fueron 45.5 casos $/ 100.000$ personas año

Edad media de diagnóstico 7 años

Síntomas: dolor abdominal, diarrea, estreñimiento, retraso del crecimiento, fatiga, irritabilidad

Enfermedades asociadas: DM-I, enfermedad tiroidea y $S D$

No se observaron variaciones de síntomas ni cambios histopatológicos durante el período estudiado

Prevalencia de EC: 28 mujeres $(51,9 \%)$ y 26 hombres (48,1\%), edad media $6.67 \pm 3.35$ años Se dividieron los pacientes en dos grupos: Los que presentaron diarrea 31 (57,4\%, 17 hombres y 14 mujeres) y los que no 23 (42,6\%, 14 mujeres y 9 hombres), éste último fueron diagnosticados a una edad posterior

Síntomas: Malnutrición (81,5\%), talla baja (87\%), raquitismo (51,9\%), uñas hipócritas (35,2\%), distensión abdominal $(57,4 \%)$. La anemia severa (2-13 años)
39

38

Suecia

Noruega el $35,5 \%$ del grupo con diarrea y $43,5 \%$ en el grupo no diarreico y la malnutrición severa en el $95,6 \%$ del grupo sin diarrea y $70,9 \%$ del grupo con diarrea.

Según la clasificación de Marsh el grado de atrofia 3b seguido de 3 c fueron los más frecuentes

El: Enfermedad inmune; GI: Gastrointestinales; ATG: Anticuerpos antitransglutaminasa tisular; SD: Síndrome Down; DH: Dermatitis herpetiforme; EMA: Anticuerpos antiendomisio; LIE: Linfocitos intraepiteliales; CoelD: Enteropatía celíaca; LymD: Síndrome Gl funcional y duodenitis linfocítica; NorD: Síndrome Gl funcional con histología duodenal normal; Ell: Enfermedad inflamatoria intestinal; EALT: Enteropatía asociada a linfoma de células T; DSG: Dieta sin gluten; NCGS: Sensibilidad al gluten no celíaca. 
Estudios clínicos sobre la enfermedad celíaca (2014-2019): revisión sistemática de la prevalencia de la presentación clínica y enfermedades asociadas por edades

(1.997 mujeres y 834 hombres) $70,5 \%$ mujeres y $29,5 \%$ hombres; total de pacientes pediátricos: 3.522 (2.139 niñas y 1.383 niños) $60,7 \%$ niñas y $39,3 \%$ niños.

En la Tabla 2 se presentan los síntomas clínicos más frecuentes durante la enfermedad celíaca.

\section{DISCUSIÓN}

Según la literatura, se observa una evolución de la forma de presentación clásica hacia la forma atípica en los últimos años. En el estudio de Rubio-Tapia et al. ${ }^{23}$ se muestra una mayor prevalencia de la EC clásica (66\%), seguida de la EC no clásica (29\%) y silente (5\%) siendo los síntomas más frecuentes diarrea, dolor abdominal y pérdida de peso. Estos resultados son similares al estudio de Dominguez et al. ${ }^{24}$, con la diferencia de una variación en la forma de presentación, observándose una disminución de los síntomas clásicos y aumento de los síntomas atípicos y asintomáticos (55,6\% EC clásica, 38,7\% EC no clásica y 5,6\% EC silente). Estudios más recientes confirman esa variación hacia la forma atípica en donde se observa el 55,7\% de EC no clásica (síntomas gastrointestinales, excepto diarrea, y extraintestinales), el 33,7\% EC clásica (síndrome de malabsorción con diarrea y pérdida de peso) y el $12 \%$ EC silente ${ }^{25}$. Otros estudios también corroboran ese incremento en las manifestaciones extradigestivas $\mathrm{s}^{22,26-28}$.

\section{Análisis de síntomas en pacientes celíacos}

Esta patología cuenta con una amplia variedad de signos y síntomas en su forma de presentación incluso muchos pacientes no manifiestan síntomas. La mayoría de los pacientes del estudio holandés (71\%) presentan 2 o más síntomas en el momento del diagnóstico, el $17,2 \%$ sólo un síntoma y el $11,7 \%$ fueron asintomáticos ${ }^{22}$; en otro estudio danés con menos participantes se muestra que el $75 \%$ de los pacientes presentó al menos una manifestación gastrointestinal (diarrea, variaciones del hábito intestinal, estreñimiento, pérdida de peso) y el $25 \%$ fueron asintomáticos ${ }^{21}$. Por lo que esta variabilidad en su forma de presentación hace difícil su diagnóstico provocando retraso en su detección.

Síntomas en personas adultas: Los síntomas que pueden presentarse en personas adultas son: diarrea, fatiga, dolor abdominal, pérdida de peso, distensión abdominal, náuseas, vómitos, dispepsia, epigastralgia, pérdida de apetito, esteatorrea, flatulencia, reflujo, irritabilidad, estreñimiento, anemia ferropénica, dermatitis herpetiforme, aftas orales, alteraciones óseas (osteopenia y osteoporosis), alteraciones psiquiátricas (ansiedad, depresión, tendencia al suicidio), alteraciones neurológicas (cefalea, epilepsia, neuropatía periférica, ataxia) y alteraciones ginecológicas (amenorrea, abortos recurrentes, menopausia precoz, infertilidad) $)^{22-24,26,28,29,31,32}$.

Los síntomas más frecuentes en las mujeres son náuseas, vómitos, estreñimiento, malestar general, fatiga, distensión

Tabla 2. Cuadro-resumen de los síntomas más frecuentes de los artículos revisados.

Síntomas típicos

\begin{tabular}{l|l|l} 
Niños y niñas & $\begin{array}{l}\text { Dolor abdominal } \\
\text { Distensión abdominal } \\
\text { Talla baja } \\
\text { Retraso en el desarrollo } \\
\text { Pérdida de peso } \\
\text { Alteraciones del metabolismo óseo } \\
\text { Anemia ferropénica }\end{array}$ \\
Adultos y adultas & $\begin{array}{l}\text { Dolor abdominal } \\
\text { Distensión abdominal } \\
\text { Anemia ferropénica } \\
\text { Diarrea } \\
\text { Pérdida de peso } \\
\text { Síndrome del intestino irritable } \\
\text { Esteatorrea } \\
\text { Espigastralgia } \\
\text { Dispepsia } \\
\text { Reflujo }\end{array}$ \\
\hline
\end{tabular}

Estreñimiento

Reflujo

Vómitos

Alteraciones cutáneas

Fatiga

Irritabilidad

Náuseas

Vómitos

Fatiga

Estreñimiento

Alteraciones cutáneas

Infertilidad

Abortos recurrentes 
abdominal y anemia; en los hombres el más frecuente la esteatorrea. La depresión, osteoporosis y fibromialgia son más frecuentes en mujeres ${ }^{23,24}$. Alrededor del $30 \%$ de los pacientes con EC presentan sobrepeso y el $12,5 \%$ obesidad en el momento del diagnóstico ${ }^{24,25,29}$. La desnutrición y el sobrepeso son dos estados muy frecuentes de la celiaquía, por lo que no debemos olvidarlos, sobre todo la obesidad/ sobrepeso que podríamos no asociar a la EC. Hay estudios que afirman que no existe una correlación estadísticamente significativa entre el valor del índice de masa corporal y el grado de atrofia vellositaria de la clasificación de Marsh $^{31}$.

Los parámetros de laboratorio que se verían alterados ante una sospecha de EC son la anemia, déficits de hierro, ferritina, folato, vitamina B12 y vitamina D. Otros marcadores observados de osteogénesis deteriorada fueron la elevación de la hormona paratiroidea (PTH) y la fosfatasa alcalina e hipocalcemia, incremento de enzimas hepáticas e hipoalbuminemia21,22,32.

La prevalencia de enfermedades autoinmunes en la EC es alrededor del 33\%, mayor en mujeres que en hombres. El $26,6 \%$ de los pacientes celíacos presentan al menos una enfermedad autoinmune, siendo más frecuentes las endocrinas $(15,8 \%)$ y dermatológicas $(11,7 \%)$, como tiroiditis autoinmune (hipotiroidismo o Graves), psoriasis, DM-I, DH, seguidas de alopecia areata, vitíligo, síndrome de Sjögren, enfermedad de Addison, liquen escleroso, hepatitis autoinmune, Ell, anemia perniciosa, artritis reumatoide, espondiloartritis, lupus eritomatoso sistémico, inflamación tejido conectivo, sarcoidosis y esclerosis múltiple. El $81,3 \%$ de los celíacos presentaron una enfermedad autoinmune y el $18,7 \%$ más de una. En el $90 \%$ de los pacientes ya se conocía la enfermedad autoinmune en el momento del diagnóstico de la EC y el resto una vez diagnosticada la EC se diagnosticó de forma concomitante otra enfermedad autoinmune. Las enfermedades autoinmunes más frecuentes en personas adultas son enfermedad tiroidea, DM-I, DH, psoriasis y El|21-25,27.

El $63,8 \%$ de los pacientes presentaron un diagnóstico previo de síndrome de intestino irritable y el 28,8\% dispepsia funcional. Los pacientes celíacos con un diagnóstico inicial de Ell fueron detectados a edades más mayores, con mayor número de visitas médicas y persistencia de síntomas durante más tiempo que aquellos pacientes sin un diagnóstico inicial de Ell. Por lo que un diagnóstico previo de síndrome de intestino irritable provoca un retraso del diagnóstico de la EC, pueden compartir síntomas ambas patologías por lo que puede llevar a errores en el diagnóstico o solaparse ambas enfermedades, provocando una reducción de la calidad de vida y aumento de la predisposición de ansiedad y depresión. Se ha observado un retraso del diagnóstico en mujeres de mediana edad, con una duración media de síntomas de unos 10 años antes del diagnóstico. Se ha observado que tras la dieta sin gluten (DSG) mejoran los síntomas y la calidad de vida de los pacientes celía $\cos ^{28}$. Los síndromes gastrointestinales (GI) funcionales cuyos síntomas más frecuentes son dolor abdominal, dispepsia, pérdida de peso y anemia, dos tercios presentan duodenitis linfocítica con recuentos elevados de LIE, por lo que al ser tan similares con la EC hay que saber distinguirlos ${ }^{27}$.

Son frecuentes las complicaciones a largo plazo si no se diagnostica la enfermedad de forma precoz, sin un adecuado diagnóstico diferencial, sino se lleva un adecuado control de la DSG o si persisten los síntomas; el 12,9\% presentan una o más complicaciones malignas, siendo el carcinoma basocelular, EALT (enteropatía asociada a linfoma de células T) y el carcinoma colorrectal los más frecuentes, observándose en mayor proporción en los hombres ${ }^{22}$.

La prevalencia de EC en familiares de primer grado de pacientes celíacos (padres, hermanos, hijos) varía ampliamente, del $9 \%$ hasta el $56 \%$, por lo que se considera un factor de riesgo y se debe realizar cribado serológico, al igual que aquellas personas con algún otro factor de riesgo como manifestaciones y enfermedades asociadas $24,25,27,28,32$. Sin embargo, la búsqueda de casos es una estrategia ineficaz para discriminar entre aquellos con y sin EC no diagnosticada, lo que plantea la cuestión de cómo detectar esta enfermedad. Hay defensores de la detección masiva, estrategia que sigue siendo controvertida, no hay pruebas suficientes sobre la rentabilidad y el beneficio de identificar y tratar la EC asintomática. Junto con los beneficios poco claros de identificar casos asintomáticos de EC, también existe la posibilidad de daños como el sobrediagnóstico, por lo que el cribado actualmente se hace en los grupos de riesgo o sospecha clara de $\mathrm{EC}^{30}$.

Síntomas en niños y niñas: Los resultados obtenidos en menores, al igual que en personas adultas, muestran un cambio en la evolución del espectro clínico de la EC (Tabla 2). En el estudio realizado por Cilleruelo et al., 2014 ${ }^{34}$, en niños y niñas de diferentes grupos de edad (0-2, >2-6, >6-15 años), se observa que la mayoría de ellos fueron diagnosticados de EC entre los 2 y 6 años (42\%), seguido del 39,5\% diagnosticados en los dos primeros años de vida y el resto $(18,4 \%)$ entre los 6-15 años. El 70,9\% presentó EC clásica, el 21,9\% EC no clásica y el $7 \%$ asintomática. La forma de presentación clásica es más frecuente en niños y niñas en edades más tempranas. Los síntomas gastrointestinales clásicos (diarrea, retraso en el desarrollo, distensión abdominal, pérdida de peso) fueron más frecuentes en menores de 6 años. En los niños y niñas mayores de 6 años predominaron los asintomáticos y síntomas no clásicos como dolor abdominal, fatiga, 
anemia ferropénica, talla baja, defectos del esmalte dental, aftas orales recurrentes, artritis, alteraciones del metabolismo óseo, epilepsia con calcificaciones cerebrales, trastornos neurológicos, DH, elevación de enzimas hepáticas. El grupo de edad de 0-2 años presentó mayor grado de desnutrición y el grupo de $>6$ años sobrepeso.

Se ha observado una disminución de niños y niñas con síntomas digestivos asociados a la EC clásica con un incrementado de síntomas extradigestivos (EC atípica) y asintomáticos (EC silente), estas dos últimas más frecuentes en niños y niñas mayores y adolescentes, por lo que a mayor edad aumentan las manifestaciones atípicas y asintomáticas. Los pacientes monosintomáticos se han incrementado en los últimos años (alrededor del 20\%) ${ }^{33-35,38}$. Los pacientes que manifiestan la forma de presentación atípica de la EC (sin diarrea) presentan una desnutrición más severa y se detecta a edades posteriores que la forma clásica o típica de la enfermedad (diarrea) ${ }^{40}$.

En niños y niñas también es frecuente el aumento de peso. El 5,8\% presentan sobrepeso y el $2,6 \%$ obesidad en el momento del diagnóstico 33,34 . El 25,7\% de los niños y niñas presentan enfermedades asociadas a la EC. Se observó una asociación en la DM-I, tiroiditis autoinmune, síndromes de Down, Turner y Williams, déficit selectivo de IgA, alergia al polen, Ell, epilepsia y familiares de primer grado. La prevalencia de enfermedades asociadas incrementa con la edad $33,34,36,37$.

El grado de atrofia vellositaria moderado-severo (Marsh III) es el más prevalente en niños y niñas, y se correlaciona con el nivel de anticuerpos ATG $\lg A^{37,40}$. Es necesario realizar un cribado serológico de celiaquía en pacientes con DM-I, a pesar de no mostrar una clínica exacerbada, porque se ha observado que al menos la mitad de los niños y niñas con DM-I en el momento del diagnóstico de la EC ya presentan atrofia vellositaria subtotal o total. Hay que tener en cuenta que el continuo daño en la mucosa intestinal provoca excesivo consumo de medicamentos, visitas médicas reiteradas y complicaciones a largo plazo. El tratamiento de la DSG en estos pacientes con EC y DM-I concomitantes podría ayudar a mantener un equilibrio adecuado de los niveles de glucemia $^{37}$. En el estudio realizado por Costa et al., 2016 ${ }^{36}$, se observó la prevalencia de EC en DM-I (4,5\%) y en síndrome de Down (13\%), con una forma de presentación de EC clásica $(32,5 \%)$, silente (5\%) y potencial $(62,5 \%)$. La forma GI prevaleció entre los casos con enfermedad celíaca activa, que se detectó en todos los niños con Síndrome de Down y en la mayoría de DM-I contribuyendo así a la porción visible del iceberg celiaco. Sin embargo la EC potencial y EC silente (la parte sumergida del iceberg) fue más prevalente en el grupo de DM-I, por lo que observamos que el perfil de la enfermedad ha cambiado después de la aparición de marcadores serológicos más sensibles y específicos, que permiten identificar pacientes con anticuerpos positivos pero aún sin hallazgos histopatológicos compatibles ${ }^{36}$.

\section{$\longrightarrow$ CONCLUSIONES}

Esta revisión permite asegurar que la prevalencia de EC se ha incrementado en los últimos años, oscilando entre el 0,4-3\%, por el mayor conocimiento de esta enfermedad, en las formas atípicas o asintomáticas y la elevada sensibilidad/especificidad en los marcadores de detección. Es más frecuente en las mujeres y puede presentarse a cualquier edad, aunque en varones jóvenes se diagnostica con menos frecuencia. A pesar de la dificultad de obtener artículos científicos con el nivel de detalle suficiente para ser incluidos en esta revisión bibliográfica, la recopilación de datos muestra cómo la forma clásica de EC predomina en la infancia, asociada frecuentemente a sintomatología digestiva (distensión y dolor abdominal, diarrea, pérdida de peso y retraso en el desarrollo). Sin embargo, en niños y niñas mayores, adolescentes y etapa adulta, son mayoritarias las formas extraintestinales o atípicas (dolor abdominal, pérdida de peso, anemia ferropénica, osteopenia, fatiga, cefalea y trastornos psiquiátricos). Se debe tener en cuenta que la celiaquía no siempre se asocia a una pérdida de peso, sino que hay pacientes que presentan un índice de masa corporal elevado con obesidad y/o sobrepeso. Es importante resaltar que se ha visto cómo en la EC son frecuentes los déficits nutricionales en el momento del diagnóstico como el déficit de hierro, vitamina $D$, folato y vitamina B12, por la alteración que provoca a nivel de la mucosa duodenal. Además, se incrementa el riesgo de padecer otras enfermedades asociadas de forma concomitante. Finalmente, señalar que el modo de presentación de la EC está cambiando, primero por un mayor nivel de conciencia sobre esta patología, pero también por cambio de los hábitos dietéticos, rápida occidentalización a nivel mundial y la mayor producción de alimentos procesados que contribuye a la exposición de antígenos de la gliadina.

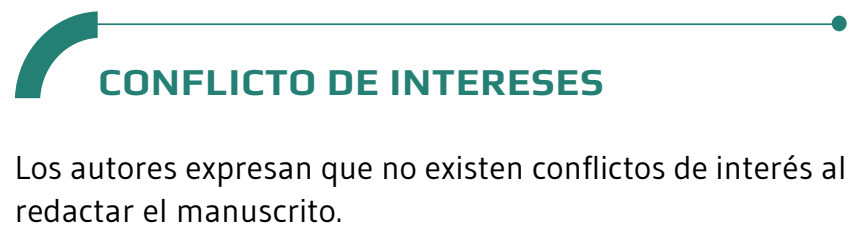




\section{REFERENCIAS}

(1) Husby S, Koletzko S, Korponay-Szabó IR, Mearin ML, Phillips A, Shamir R, et al. European Society for Pediatric Gastroenterology, Hepatology, and Nutrition guidelines for the diagnosis of coeliac disease. J Pediatr Gastroenterol Nutr. 2012; 54(1): 136-60.

(2) Elli L, Ferretti F, Orlando S, Vecchi M, Monguzzi E, Roncoroni $\mathrm{L}$, et al. Management of celiac disease in daily clinical practice. Eur J Intern Med. 2019; 61: 15-24.

(3) Hurley JJ, Lee B, Turner JK, Beale A, Jenkins HR, Swift GL. Incidence and presentation of reported coeliac disease in Cardiff and the Vale of Glamorgan: the next 10 years. Eur ] Gastroenterol Hepatol. 2012; 24(5): 482-6.

(4) Dixit R, Lebwohl B, Ludvigsson JF, Lewis SK, Rizkalla-Reilly $N$, Green PHR. Celiac disease is diagnosed less frequently in young adult males. Dig Dis Sci. 2014; 59(7): 1509-12.

(5) van Gils T, Rootsaert B, Bouma G, Mulder CJ]. Celiac Disease in The Netherlands: Demographic Data of Members of the Dutch Celiac Society. J Gastrointestin Liver Dis. 2016; 25(4): 441-5.

(6) Glissen Brown JR, Singh P. Coeliac disease. Paediatr Int Child Health. 2019; 39(1): 23-31.

(7) Van Kalleveen MW, de Meij T, Plötz FB. Clinical spectrum of paediatric coeliac disease: a 10-year single-centre experience. Eur J Pediatr. 2018; 177(4): 593-602.

(8) Akman S, Şahaloğlu Ö, Dalkan C, Bahçeciler NN, Arıkan Ç. Is celiac disease misdiagnosed in children with functional constipation? Turk J Gastroenterol. 2018; 29(2): 210-4.

(9) de Carvalho FK, de Queiroz AM, da Silva RAB, Sawamura R, Bachmann L, da Silva LAB, Nelson-Filho P. Oral aspects in celiac disease children: clinical and dental enamel chemical evaluation. Oral Surg Oral Med Oral Pathol Oral Radiol Endod. 2015; 119(6): 636-43.

(10) Bashiri H, Afshari D, Babaei N, Ghadami MR. Celiac Disease and Epilepsy: The Effect of Gluten-Free Diet on Seizure Control. Adv Clin Exp Med. 2016; 25(4): 751-4.

(11) Zis P, Julian T, Hadjivassiliou M. Headache Associated with Coeliac Disease: A Systematic Review and Meta-Analysis. Nutrients. 2018; 10(10).

(12) Mahadev S, Laszkowska M, Sundström J, Björkholm M, Lebwohl B, Green PHR, et al. Prevalence of Celiac Disease in Patients With Iron Deficiency Anemia-A Systematic Review With Meta-analysis. Gastroenterol. 2018; 155(2): 374-382.e1.

(13) Hujoel IA, Reilly NR, Rubio-Tapia A. Celiac Disease: Clinical Features and Diagnosis. Gastroenterol Clin North Am. 2019; 48(1): 19-37.

(14) López Casado MÁ, Lorite P, Ponce de León C, Palomeque T, Torres Ml. Celiac Disease Autoimmunity. Arch Immunol Ther Exp (Warsz). 2018; 66(6): 423-30.

(15) Mellander M-R, Ekbom A, Hultcrantz R, Löfberg R, Öst Å, Björk J. Microscopic colitis: a descriptive clinical cohort study of 795 patients with collagenous and lymphocytic colitis. Scand J Gastroenterol. 2016; 51(5): 556-62.

(16) Dereci S, Pirgon O, Gürsoy Koca T, Akcam M. Celiac disease in an obese child with down syndrome. 2017. 2015; 34(2): 133-5.

(17) Lindfors K, Ciacci C, Kurppa K, Lundin KEA, Makharia GK, Mearin
ML, et al. Coeliac disease. Nat Rev Dis Primers. 2019; 5(1): 3.

(18) Ludvigsson JF, Lebwohl B, Green PHR, Chung WK, Mårild K. Celiac disease and Down syndrome mortality: a nationwide cohort study. BMC Pediatr. 2017; 17(1): 41.

(19) Román Riechmann E, Cilleruelo Pascual ML, Gutiérrez Junquera $\mathrm{Cl}$. Epidemiología de la enfermedad celíaca. Polanco Allué isabel, editor. Madrid: Ergón; 2013.

(20) Moher D, Liberati A, Tetzlaff J, Altman DG, PRISMA Group. Preferred reporting items for systematic reviews and metaanalyses: the PRISMA statement. Ann Intern Med. 2009; 151(4): 264-9.

(21) Schøsler L, Christensen LA, Hvas CL. Symptoms and findings in adult-onset celiac disease in a historical Danish patient cohort. Scand J Gastroenterol. 2016; 51(3): 288-94.

(22) Spijkerman M, Tan IL, Kolkman J], Withoff S, Wijmenga C, Visschedijk MC, et al. A large variety of clinical features and concomitant disorders in celiac disease - A cohort study in the Netherlands. Dig Liver Dis. 2016; 48(5): 499-505.

(23) Rubio-Tapia A, Jansson-Knodell CL, Rahim MW, See JA, Murray JA. Influencia del género en la presentación clínica y enfermedades asociadas en adultos con enfermedad celíaca (EC). Gac Med Mex. 2016; 152(S2): 38-46.

(24) Dominguez Castro P, Harkin G, Hussey M, Christopher B, Kiat C, Liong Chin J, et al. Changes in Presentation of Celiac Disease in Ireland From the 1960s to 2015. Clin Gastroenterol Hepatol. 2017; 15(6): 864-71.

(25) Conti L, Lahner E, Galli G, Esposito G, Carabotti M, Annibale B. Risk Factors Associated with the Occurrence of Autoimmune Diseases in Adult Coeliac Patients. Gastroenterol Res Pract. 2018: 3049286.

(26) Mooney PD, Leeds JS, Libzo N, Sidhu R, Evans KE, Hall EJ, et al. Case-finding for coeliac disease in secondary care: a prospective multicentre UK study. Dig Liver Dis. 2014; 46(1): 32-5.

(27) Zanini B, Lanzarotto F, Villanacci V, Carabellese N, Ricci C, Lanzini A. Clinical expression of lymphocytic duodenosis in "mild enteropathy" celiac disease and in functional gastrointestinal syndromes. Scand ] Gastroenterol. 2014; 49(7): 794-800.

(28) Ramírez-Cervantes KL, Remes-Troche JM, Del Pilar MilkeGarcía M, Romero V, Uscanga LF. Characteristics and factors related to quality of life in Mexican Mestizo patients with celiac disease. BMC Gastroenterol. 2015; 15: 4.

(29) Ribeiro PV de M, Santos A de P, Andreoli CS, Ribeiro SMR, Jorge $M$ de $P$, Moreira AVB. Nutritional status variation and intestinal and extra intestinal symptomatology in patients with celiac disease and non-celiac gluten sensitivity given specialized dietary advice. Rev Nutr. 2017; 30(1): 57-67.

(30) Hujoel IA, Van Dyke CT, Brantner T, Larson J, King KS, Sharma A, et al. Natural history and clinical detection of undiagnosed coeliac disease in a North American community. Aliment Pharmacol Ther. 2018; 47(10): 1358-66.

(31) Maxim R, Pleşa A, Ciortescu I, Gîrleanu I, Stoica O, Trifan A-V. Celiac disease: 10-year experience in a Romanian tertiary center. Rev Med Chir Soc Med Nat lasi. 2014; 118(4): 986-91.

(32) Nousseiba Bellir, Mohamed Nacer Bellir, Leila Rouabah. Celiac disease in adults: Report of 73 cases features of eastern Algeria. 2014. 27(1): 224-7. 
(33) Gokce S, Arslantas E. Changing face and clinical features of celiac disease in children. Pediatr Int. 2015; 57(1): 107-12.

(34) Cilleruelo ML, Roman-Riechmann E, Sanchez-Valverde F, Donat E, Manuel-Ramos J, Martín-Orte E, et al. Spanish national registry of celiac disease: incidence and clinical presentation. ] Pediatr Gastroenterol Nutr. 2014; 59(4): 522-6.

(35) Khatib M, Baker RD, Ly EK, Kozielski R, Baker SS. Presenting Pattern of Pediatric Celiac Disease. ] Pediatr Gastroenterol Nutr. 2016; 62(1): 60-3.

(36) Costa Gomes R, Cerqueira Maia J, Fernando Arrais R, André Nunes Jatobá C, Auxiliadora Carvalho Rocha M, Edinilma Felinto Brito $M$, et al. The celiac iceberg: from the clinical spectrum to serology and histopathology in children and adolescents with type 1 diabetes mellitus and Down syndrome. Scand J Gastroenterol. 2016; 51(2): 178-85.
(37) Laitinen AU, Agardh D, Kivelä L, Huhtala H, Lähdeaho M-L, Kaukinen $\mathrm{K}$, et al. Coeliac patients detected during type 1 diabetes surveillance had similar issues to those diagnosed on a clinical basis. Acta Paediatr. 2017; 106(4): 639-46.

(38) Tapsas D, Hollén E, Stenhammar L, Fälth-Magnusson K. The clinical presentation of coeliac disease in 1030 Swedish children: Changing features over the past four decades. Dig Liver Dis. 2016; 48(1): 16-22.

(39) Beitnes A-CR, Vikskjold FB, Jóhannesdóttir GB, Perminow G, Olbjørn C, Andersen SN, et al. Symptoms and Mucosal Changes Stable During Rapid Increase of Pediatric Celiac Disease in Norway. J Pediatr Gastroenterol Nutr. 2017; 64(4): 586-91.

(40) Hashmi MA, Hussain T, Masood N, Asghar RM. Diarrheal Versus Non-diarrheal Presentations of Paediatric Celiac Disease. ] Coll Physicians Surg Pak. 2016; 26(8): 662-6. 